\title{
LETTER
}

\section{Inhaled amphotericin B lipid complex for prophylaxis against COVID-19-associated invasive pulmonary aspergillosis}

\author{
María Cruz Soriano ', Gabriela Narváez-Chávez ${ }^{1}$, Marina López-Olivencia', Jesús Fortún² and Raúl de Pablo ${ }^{3^{*}}$ (1)
}

(c) 2021 Springer-Verlag GmbH Germany, part of Springer Nature

\section{Dear Editor,}

Recently, Prattes and colleagues [1] published in Intensive Care Medicine a high incidence of coronavirus disease 2019-associated invasive pulmonary aspergillosis (CAPA), according to 2020 ECMM/ISHAM consensus criteria [2], with a correspondingly high mortality rate. However, both the guidelines and a Taskforce report published recently [3] only considered host risk factors [3]. Many work-related aspergillosis outbreaks have been reported due to airborne contamination with Aspergillus spores [4]. Protective measures against environmental contamination must be taken when there is construction activity on-site in a hospital. Antifungal prophylaxis is not recommended in mechanical ventilated patients affected by coronavirus disease 2019 (COVID-19) [3].

We implemented an active surveillance protocol for CAPA in mechanically ventilated patients with COVID19 admitted to the intensive care unit (ICU). The protocol consisted of weekly assessment of galactomannan (GM), fungal culture, and calcofluor white microscopy from endotracheal aspirate for all patients. If test results were positive (GM cutoff $>0.9$ ) or the patient suffered respiratory deterioration, a bronchoscopy with bronchoalveolar lavage (BAL) was performed. CAPA case definition utilized was in accordance with ECMM/ISHAM consensus criteria [2].

In April 2021, there was a CAPA outbreak within the ICU which was associated with remodeling carried out one floor below, despite protection measures having

*Correspondence: raul.depablo.uah@gmail.com

${ }^{3}$ Intensive Care Department, Hospital Universitario Ramón y Cajal, Universidad de Alcalá, IRYCIS, Madrid, Spain

Full author information is available at the end of the article been implemented. Eleven patients developed CAPA in 3 weeks, which represented an incidence of $22.4 \%$ (11/49). The predominant microbiological diagnostic tool was a BAL GM, which was positive in $90 \%$ of patients. In $72 \%$, both culture and BAL GM were positive. Air environmental measurements were performed and levels higher than $10 \mathrm{cfu} / \mathrm{m}^{3}$ were considered positive.

In response to the outbreak, protective measures were intensified. All mechanically ventilated patients received $50 \mathrm{mg}$ every $48 \mathrm{~h}$ of inhaled amphotericin B lipid complex (ABLC). The isolation rooms were cleaned and closed, and additional cleaning of workspaces and hallways was performed, with special emphasis on architectural isolation of the renovation work. Despite the persistence of airborne Aspergillus contamination during 34 consecutive days, no patient who received ABLC developed CAPA (Fig. 1). Interestingly, only two patients with bronchospasm, who did not receive $\mathrm{ABCL}$, developed CAPA. The patients' characteristics, ICU pressure, and treatment over time did not change. A total of 45 patients received prophylaxis with inhaled ABLC. Of these, four $(8.8 \%)$ suffered bronchospasm and in one patient, the effect was severe enough that a determination was made to suspend the prophylaxis. An additional $33.3 \%(15 / 45)$ experienced mechanical problems due to the buildup of the drug in the filter of the expiratory limb of the ventilator, but in all cases the issue was able to be solved by the nursing staff and caused no clinical impact on the patients.

We conclude that the application of a set of increased environmental protective measures and supplementary inhaled ABCL should be considered to control an outbreak of CAPA in mechanically ventilated patients with COVID-19. We agree with Koehler and colleagues [2],

\section{Springer}




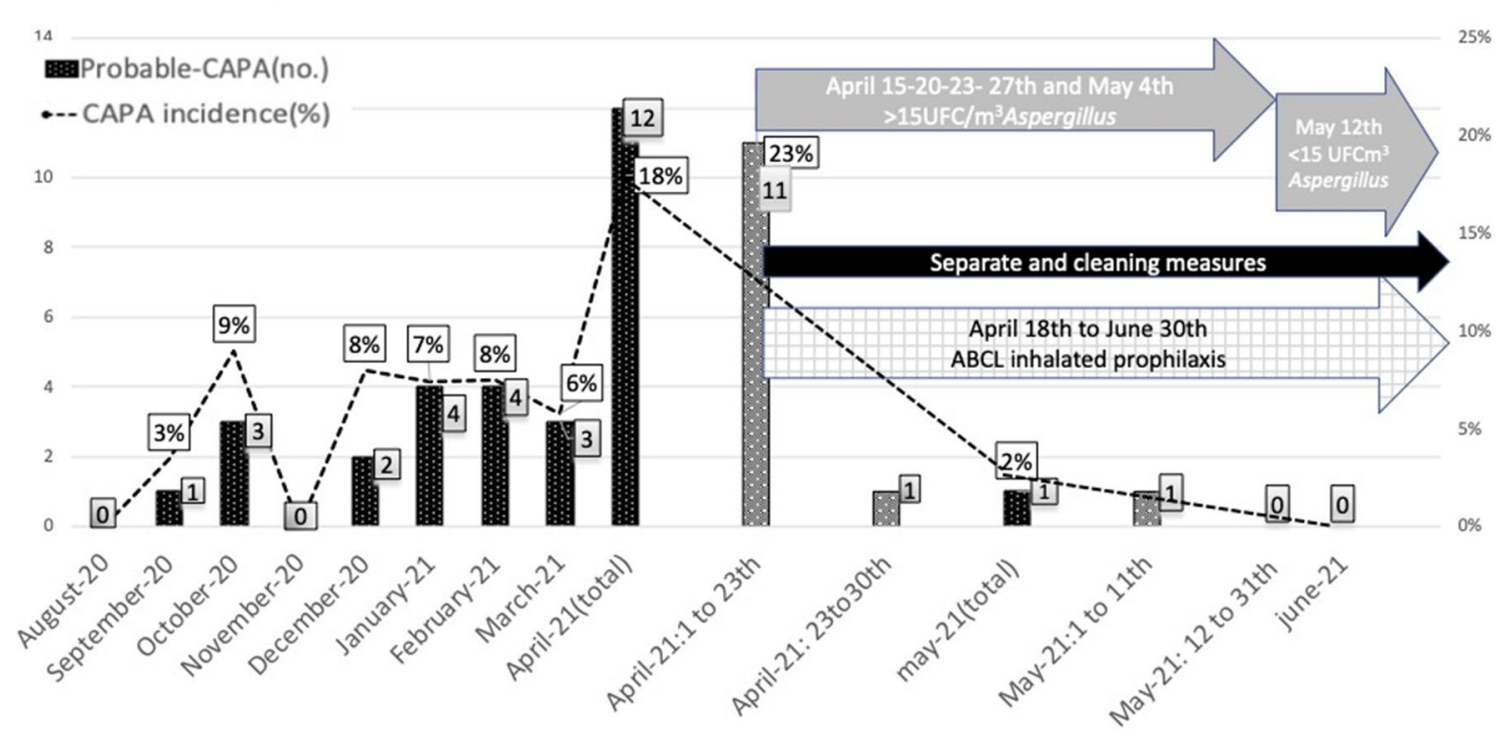

Fig. 1 CAPA diagnosis from August 2020 to June 2021

who suggest that the reported excess mortality might justify antifungal prophylaxis trials, similar to those proposed for patients with invasive aspergillosis [5]. Therefore, inhaled antifungal prophylaxis may be an effective option to reduce CAPA when its incidence is elevated.

\section{Author details}

${ }^{1}$ Intensive Care Department, Hospital Universitario Ramón y Cajal, Universidad de Alcalá, Carretera de Colmena Viejo, km 9,100, 28034 Madrid, Spain. ${ }^{2}$ Infectious Diseases Department, Hospital Ramón y Cajal, Instituto Ramón y Cajal de Investigación Sanitaria (IRYCIS), Madrid, Spain. ${ }^{3}$ Intensive Care Department, Hospital Universitario Ramón y Cajal, Universidad de Alcalá, IRYCIS, Madrid, Spain.

\section{Acknowledgements}

We want to thank Preventive Medicine Service, Department of Microbiology and Pharmacy Service, Hospital Ramon y Cajal, Madrid, Spain and our Intensive Care nursery staff for their enormous labor.

\section{Declarations}

\section{Conflicts of interest}

The authors declare that they have no conflicts of interest.

\section{Ethics approval}

The study protocol was approved by the institutional Ethics and Clinical Research Committee.

\section{Publisher's Note}

Springer Nature remains neutral with regard to jurisdictional claims in published maps and institutional affiliations.

Accepted: 3 December 2021

Published online: 23 December 2021

\section{References}

1. Prattes J, Wauters J, Giacobbe DR et al (2021) Diagnosis and treatment of COVID-19 associated pulmonary apergillosis in critically ill patients: results from a European confederation of medical mycology registry. Intensive Care Med. https://doi.org/10.1007/s00134-021-06471-6

2. Koehler P, Bassetti M, Chakrabarti A et al (2021) Defining and managing COVID-19-associated pulmonary aspergillosis: the 2020 ECMM/ISHAM consensus criteria for research and clinical guidance. Lancet Infect Dis 21:e149-e162. https://doi.org/10.1016/S1473-3099(20)30847-1

3. Verweij PE, Brüggemann RJM, Azoulay E et al (2021) Taskforce report on the diagnosis and clinical management of COVID-19 associated pulmonary aspergillosis. Intensive Care Med 47:819-834. https://doi.org/ 10.1007/s00134-021-06449-4

4. Fournel I, Sautour M, Lafon I et al (2010) Airborne aspergillus contamination during hospital construction works: efficacy of protective measures. Am J Infect Control 38:189-194. https://doi.org/10.1016/j.ajic.2009.07.011

5. Verweij PE, Rijnders BJA, Brüggemann RJM et al (2020) Review of influenza-associated pulmonary aspergillosis in ICU patients and proposal for a case definition: an expert opinion. Intensive Care Med 46:1524-1535. https://doi.org/10.1007/s00134-020-06091-6 